\title{
Correction to: Knock-Down of CD24 in Astrocytes Aggravates Oxyhemoglobin-Induced Hippocampal Neuron Impairment
}

\author{
Xiang-Xin Chen ${ }^{1} \cdot$ Tao Tao $^{2} \cdot$ Sen Gao ${ }^{1} \cdot$ Han Wang ${ }^{1,3} \cdot$ Xiao-Ming Zhou $^{4} \cdot$ Yong-Yue Gao ${ }^{1} \cdot$ Chun-Hua Hang ${ }^{1} \cdot$ Wei Li $^{1}$
}

Published online: 6 January 2022

○) Springer Science+Business Media, LLC, part of Springer Nature 2022

\section{Correction to: Neurochemical Research https://doi.org/10.1007/s1 1064-021-03468-x}

In the original version of this article, the given and family names of all the authors were incorrectly structured. The correct structure of the author names is "Xiang-Xin Chen, Tao Tao, Sen Gao, Han Wang, Xiao-Ming Zhou, Yong-Yue Gao, Chun-Hua Hang, Wei Li”.

The original article has been corrected.

Publisher's Note Springer Nature remains neutral with regard to jurisdictional claims in published maps and institutional affiliations.

Xiang-Xin Chen and Tao Tao have contributed equally to this work.

The original article can be found online at https://doi.org/10.1007/ s11064-021-03468-x.

Chun-Hua Hang

hang_neurosurgery@ 163.com

$\triangle$ Wei Li

lwxzlw@126.com

1 Department of Neurosurgery, Nanjing Drum Tower Hospital, The Affiliated Hospital of Nanjing University Medical School, 321 Zhongshan Road, Nanjing 210008, China

2 Department of Neurosurgery, Nanjing Drum Tower Hospital, Clinical College of Nanjing Medical University, Nanjing, China

3 Department of Neurosurgery, The First School of Medicine, Jinling Hospital, Southern Medicine University (Guangzhou), Nanjing, China

4 Department of Neurosurgery, School of Medicine, Jinling Hospital, Nanjing University, Nanjing, China 